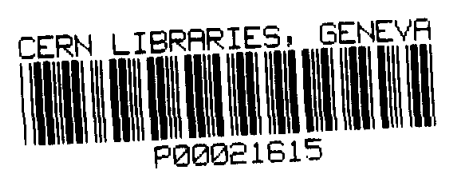

CERN-PPE/94-30

23 February 1994

$\operatorname{sen} 9410$

\title{
Z Production Cross Sections and Lepton Pair Forward-Backward Asymmetries
}

The ALEPH Collaboration ${ }^{1}$

\begin{abstract}
Precise values of the $\mathrm{Z}$ resonance cross sections and lepton pair forwardbackward asymmetries are measured from a sample of 1.3 million $Z$ decays into hadrons and charged leptons taken with the ALEPH detector at LEP. A silicon-tungsten luminosity calorimeter was installed in ALEPH in 1992, permitting the determination of the absolute luminosity to a systematic precision of $0.15 \%$ (experimental) and $0.25 \%$ (theoretical). This is combined with a better understanding of the hadronic event selection and an improved tau pair selection. Including the ALEPH results of the past three years one obtains $\sigma_{h}^{0}=(41.60 \pm 0.16) \mathrm{nb}$, and $\mathrm{R}_{l}=20.69 \pm 0.09$. The corresponding number of light neutrino species is $N_{\nu}=2.983 \pm 0.034$. The lepton pair forward-backward asymmetry is used to derive the ratio of vector to axial-vector couplings of leptons: $g_{V}^{2}\left(M_{Z}^{2}\right) / g_{A}^{2}\left(M_{Z}^{2}\right)=0.0058 \pm 0.0011$. An application of these results to constrain one model with extra $Z$ bosons is presented.
\end{abstract}

(Submitted to Z. Phys. C)

\footnotetext{
${ }^{1}$ See the following pages for the list of authors.
} 


\section{The ALEPH Collaboration}

D. Buskulic, D. Casper, I. De Bonis, D. Decamp, P. Ghez, C. Goy, J.-P. Lees, M.-N. Minard, P. Odier, B. Pietrzyk

Laboratoire de Physique des Particules (LAPP), IN ${ }^{2} P^{3}$-CNRS, 74019 Annecy-le-Vieux Cedex, France

F. Ariztizabal, P. Comas, J.M. Crespo, I. Efthymiopoulos, E. Fernandez, M. Fernandez-Bosman, V. Gaitan, Ll. Garrido, ${ }^{29}$ M. Martinez, T. Mattison, ${ }^{30}$ S. Orteu, A. Pacheco, C. Padilla, A. Pascual

Institut de Fisica d'Altes Energies, Universitat Autonoma de Barcelona, 08193 Bellaterra (Barcelona), Spain ${ }^{7}$

D. Creanza, M. de Palma, A. Farilla, G. Iaselli, G. Maggi, N. Marinelli, S. Natali, S. Nuzzo, A. Ranieri, G. Raso, F. Romano, F. Ruggieri, G. Selvaggi, L. Silvestris, P. Tempesta, G. Zito

Dipartimento di Fisica, INFN Sezione di Bari, 70126 Bari, Italy

Y. Chai, D. Huang, X. Huang, J. Lin, T. Wang, Y. Xie, D. Xu, R. Xu, J. Zhang, L. Zhang, W. Zhao Institute of High-Energy Physics, Academia Sinica, Beijing, The People's Republic of China ${ }^{8}$

G. Bonvicini, J. Boudreau, ${ }^{25}$ H. Drevermann, R.W. Forty, G. Ganis, C. Gay, ${ }^{3}$ M. Girone, R. Hagelberg, J. Harvey, J. Hilgart, ${ }^{27}$ R. Jacobsen, B. Jost, J. Knobloch, I. Lehraus, M. Maggi, C. Markou, P. Mato, H. Meinhard, A. Minten, R. Miquel, K. Moffeit, P. Palazzi, J.R. Pater, J.A. Perlas, P. Perrodo, J.F. Pusztaszeri, F. Ranjard, L. Rolandi, J. Rothberg, ${ }^{2}$ T. Ruan, M. Saich, D. Schlatter, M. Schmelling, F. Sefkow, ${ }^{6}$ W. Tejessy, I.R. Tomalin, R. Veenhof, H. Wachsmuth, S. Wasserbaech, ${ }^{2}$ W. Wiedenmann, T. Wildish, W. Witzeling, J. Wotschack

European Laboratory for Particle Physics (CERN), 1211 Geneva 23, Switzerland

Z. Ajaltouni, M. Bardadin-Otwinowska, A. Barres, C. Boyer, A. Falvard, P. Gay, C. Guicheney, P. Henrard, J. Jousset, B. Michel, J-C. Montret, D. Pallin, P. Perret, F. Podlyski, J. Proriol, F. Saadi

Laboratoire de Physique Corpusculaire, Université Blaise Pascal, IN ${ }^{2} P^{3}-C N R S$, ClermontFerrand, 63177 Aubière, France

T. Fearnley, J.B. Hansen, J.D. Hansen, J.R. Hansen, P.H. Hansen, S.D. Johnson, R. Møllerud, B.S. Nilsson ${ }^{1}$

Niels Bohr Institute, 2100 Copenhagen, Denmark ${ }^{9}$

A. Kyriakis, E. Simopoulou, I. Siotis, A. Vayaki, K. Zachariadou

Nuclear Research Center Demokritos (NRCD), Athens, Greece

J. Badier, A. Blondel, G. Bonneaud, J.C. Brient, P. Bourdon, G. Fouque, L. Passalacqua, A. Rougé, M. Rumpf, R. Tanaka, M. Verderi, H. Videau

Laboratoire de Physique Nucléaire et des Hautes Energies, Ecole Polytechnique, $I N^{2} P^{3}$ CNRS, 91128 Palaiseau Cedex, France 
D.J. Candlin, M.I. Parsons, E. Veitch

Department of Physics, University of Edinburgh, Edinburgh EH9 3JZ, United Kingdom ${ }^{10}$

E. Focardi, L. Moneta, G. Parrini

Dipartimento di Fisica, Università di Firenze, INFN Sezione di Firenze, 50125 Firenze, Italy

M. Corden, M. Delfino, ${ }^{12}$ C. Georgiopoulos, D.E. Jaffe, D. Levinthal ${ }^{15}$

Supercomputer Computations Research Institute, Florida State University, Tallahassee, FL $32306-4052$, USA $^{13,14}$

A. Antonelli, G. Bencivenni, G. Bologna, ${ }^{4}$ F. Bossi, P. Campana, G. Capon, F. Cerutti, V. Chiarella, G. Felici, P. Laurelli, G. Mannocchi, ${ }^{5}$ F. Murtas, G.P. Murtas, M. Pepe-Altarelli, S. Salomone

Laboratori Nazionali dell'INFN (LNF-INFN), 00044 Frascati, Italy

P. Colrain, I. ten Have, I.G. Knowles, J.G. Lynch, W. Maitland, W.T. Morton, C. Raine, P. Reeves, J.M. Scarr, K. Smith, M.G. Smith, A.S. Thompson, S. Thorn, R.M. Turnbull

Department of Physics and Astronomy, University of Glasgow, Glasgow G12 8QQ,United Kingdom ${ }^{10}$

U. Becker, O. Braun, C. Geweniger, P. Hanke, V. Hepp, E.E. Kluge, A. Putzer, ${ }^{1}$ B. Rensch, M. Schmidt H. Stenzel, K. Tittel, M. Wunsch

Institut für Hochenergiephysik, Universität Heidelberg, 69120 Heidelberg, Fed. Rep. of Germany ${ }^{16}$

R. Beuselinck, D.M. Binnie, W. Cameron, M. Cattaneo, D.J. Colling, P.J. Dornan, J.F. Hassard, N. Konstantinidis, A. Moutoussi, J. Nash, D.G. Payne, G. San Martin, J.K. Sedgbeer, A.G. Wright

Department of Physics, Imperial College, London SW7 2BZ, United Kingdom ${ }^{10}$

P. Girtler, D. Kuhn, G. Rudolph, R. Vogl

Institut für Experimentajphysik, Universität Innsbruck, 6020 Innsbruck, Austria ${ }^{18}$

C.K. Bowdery, T.J. Brodbeck, A.J. Finch, F. Foster, G. Hughes, D. Jackson, N.R. Keemer, M. Nuttall, A. Patel, T. Sloan, S.W. Snow, E.P. Whelan

Department of Physics, University of Lancaster, Lancaster LA1 4YB, United Kingdom ${ }^{10}$

A. Galla, A.M. Greene, K. Kleinknecht, J. Raab, B. Renk, H.-G. Sander, H. Schmidt, S.M. Walther, R. Wanke, B. Wolf

Institut für Physik, Universität Mainz, 55099 Mainz, Fed. Rep. of Germany ${ }^{16}$

A.M. Bencheikh, C. Benchouk, A. Bonissent, D. Calvet, J. Carr, P. Coyle, C. Diaconu, F. Etienne, D. Nicod, P. Payre, L. Roos, D. Rousseau, P. Schwemling, M. Talby

Centre de Physique des Particules, Faculté des Sciences de Luminy, IN²P3_CNRS, 13288 Marseille, France 
S. Adlung, R. Assmann, C. Bauer, W. Blum, D. Brown, P. Cattaneo, ${ }^{23}$ B. Dehning, H. Dietl, F. Dydak, ${ }^{21}$ M. Frank, A.W. Halley, K. Jakobs, J. Lauber, G. Lütjens, G. Lutz, W. Männer, H.-G. Moser, R. Richter, J. Schröder, A.S. Schwarz, R. Settles, H. Seywerd, U. Stierlin, U. Stiegler, R. St. Denis, G. Wolf Max-Planck-Institut für Physik, Werner-Heisenberg-Institut, 80805 München, Fed. Rep. of Germany ${ }^{16}$

R. Alemany, J. Boucrot, ${ }^{1}$ O. Callot, A. Cordier, M. Davier, L. Duflot, J.-F. Grivaz, Ph. Heusse, P. Janot, D.W. Kim ${ }^{19}$ F. Le Diberder, J. Lefrançois, A.-M. Lutz, G. Musolino, M.-H. Schune, J.-J. Veillet, I. Videau

Laboratoire de l'Accélérateur Linéaire, Université de Paris-Sud, IN² $P^{3}$-CNRS, 91405 Orsay Cedex, France

D. Abbaneo, G. Bagliesi, G. Batignani, U. Bottigli, C. Bozzi, G. Calderini, M. Carpinelli, M.A. Ciocci, V. Ciulli, R. Dell'Orso, I. Ferrante, F. Fidecaro, L. Foà, ${ }^{1}$ F. Forti, A. Giassi, M.A. Giorgi, A. Gregorio, F. Ligabue, A. Lusiani, P.S. Marrocchesi, E.B. Martin, A. Messineo, F. Palla, G. Rizzo, G. Sanguinetti, P. Spagnolo, J. Steinberger, R. Tenchini, ${ }^{1}$ G. Tonelli, ${ }^{28}$ G. Triggiani, A. Valassi, C. Vannini, A. Venturi, P.G. Verdini, J. Walsh

Dipartimento di Fisica dell'Università, INFN Sezione di Pisa, e Scuola Normale Superiore, 56010 Pisa, Italy

A.P. Betteridge, Y. Gao, M.G. Green, D.L. Johnson, P.V. March, T. Medcalf, LI.M. Mir, I.S. Quazi, J.A. Strong

Department of Physics, Royal Holloway \& Bedford New College, University of London, Surrey TW20 OEX, United Kingdom ${ }^{10}$

V. Bertin, D.R. Botterill, R.W. Clifft, T.R. Edgecock, S. Haywood, M. Edwards, P.R. Norton, J.C. Thompson

Particle Physics Dept., Rutherford Appleton Laboratory, Chilton, Didcot, Oxon OX11 OQX, United Kingdom ${ }^{10}$

B. Bloch-Devaux, P. Colas, H. Duarte, S. Emery, W. Kozanecki, E. Lançon, M.C. Lemaire, E. Locci, B. Marx, P. Perez, J. Rander, J.-F. Renardy, A. Rosowsky, A. Roussarie, J.-P. Schuller, J. Schwindling, D. Si Mohand, B. Vallage

Service de Physique des Particules, DAPNIA, CE-Saclay, 91191 Gif-sur-Yvette Cedex, France $^{17}$

R.P. Johnson, A.M. Litke, G. Taylor, J. Wear

Institute for Particle Physics, University of California at Santa Cruz, Santa Cruz, CA 95064, $U_{S A^{22}}$

W. Babbage, E. Beddall C.N. Booth, S. Cartwright, F. Combley, I. Dawson, C. Rankin, L.F. Thompson Department of Physics, University of Sheffield, Sheffield S3 7RH, United Kingdom ${ }^{10}$

A. Böhrer, S. Brandt, G. Cowan, ${ }^{1}$ E. Feigl, C. Grupen, G. Lutters, J. Minguet-Rodriguez, F. Rivera, ${ }^{26}$ P. Saraiva, U. Schäfer, L. Smolik

Fachbereich Physik, Universität Siegen, 57068 Siegen, Fed. Rep. of Germany ${ }^{16}$ 
L. Bosisio, R. Della Marina, G. Giannini, B. Gobbo, L. Pitis, F. Ragusa ${ }^{20}$

Dipartimento di Fisica, Unjversità di Trieste e INFN Sezione di Trieste, 34127 Trieste, Italy

L. Bellantoni, W. Chen, J.S. Conway, ${ }^{24}$ Z. Feng, D.P.S. Ferguson, Y.S. Gao, J. Grahl, J.L. Harton, O.J. Hayes, H. Hu, J.M. Nachtman, Y.B. Pan, Y. Saadi, M. Schmitt, I. Scott, V. Sharma, J.D. Turk, A.M. Walsh, F.V. Weber, Sau Lan Wu, X. Wu, M. Zheng, J.M. Yamartino, G. Zobernig

Department of Physics, University of Wisconsin, Madison, WI 53706, USA ${ }^{11}$

\footnotetext{
${ }^{1}$ Now at CERN, PPE Division, 1211 Geneva 23, Switzerland.

${ }^{2}$ Permanent address: University of Washington, Seattle, WA 98195 , USA.

${ }^{3}$ Now at Harvard University, Cambridge, MA 02138, U.S.A.

${ }^{4}$ Also Istituto di Fisica Generale, Università di Torino, Torino, Italy.

${ }^{5}$ Also Istituto di Cosmo-Geofisica del C.N.R., Torino, Italy.

${ }^{6}$ Now at DESY, Hamburg, Germany.

${ }^{7}$ Supported by CICYT, Spain.

${ }^{8}$ Supported by the National Science Foundation of China.

${ }^{9}$ Supported by the Danish Natural Science Research Council.

${ }^{10}$ Supported by the UK Science and Engineering Research Council.

${ }^{11}$ Supported by the US Department of Energy, contract DE-AC02-76ER00881.

${ }^{12}$ On leave from Universitat Autonoma de Barcelona, Barcelona, Spain.

${ }^{13}$ Supported by the US Department of Energy, contract DE-FG05-92ER40742.

${ }^{14}$ Supported by the US Department of Energy, contract DE-FC05-85ER250000.

${ }^{15}$ Present address: Lion Valley Vineyards, Cornelius, Oregon, U.S.A.

${ }^{16}$ Supported by the Bundesministerium für Forschung und Technologie, Fed. Rep. of Germany.

${ }^{17}$ Supported by the Direction des Sciences de la Matière, C.E.A.

${ }^{18}$ Supported by Fonds zur Förderung der wissenschaftlichen Forschung, Austria.

${ }^{19}$ Permanent address: Kangnung National University, Kangnung, Korea.

${ }^{20}$ Now at Dipartimento di Fisica, Università di Milano, Milano, Italy.

${ }^{21}$ Also at CERN, PPE Division, 1211 Geneva 23, Switzerland.

${ }^{22}$ Supported by the US Department of Energy, grant DE-FG03-92ER40689.

${ }^{23}$ Now at Università di Pavia, Pavia, Italy.

${ }^{24}$ Now at Rutgers University, Piscataway, NJ 08854, USA.

${ }^{25}$ Now at FERMILAB, Batavia, IL 60510, U.S.A.

${ }^{26}$ Partially supported by Colciencias, Colombia.

${ }^{27}$ Now at SSCL, Dallas 75237-3946, TX, U.S.A.

${ }^{28}$ Also at Istituto di Matematica e Fisica, Università di Sassari, Sassari, Italy.

${ }^{29}$ Permanent address: Dept. d'Estructura i Constituents de la Materia, Universitat de Barcelona, 08208 Barcelona, Spain.

${ }^{30}$ Now at SLAC, Stanford, CA 94309, U.S.A.
} 


\section{Introduction}

The precision of the absolute cross section measurements in ALEPH has been improved with the installation in September 1992 of a silicon-tungsten luminosity monitor (SiCAL). Additional improvements over previous ALEPH publications [1,2] come from a re-evaluation of the systematic error in the hadron selection and an improved tau-pair selection. Only the differences between the present analysis and that previously published are described in detail.

The ALEPH detector has been described elsewhere [3]. The SiCAL upgrade introduced two luminosity setups for the measurements in 1992 for which the data were analyzed separately:

1. LCAL period: luminosity measurements up to the September 1992 LEP technical stop were performed with the lead-proportional wire chamber calorimeter (LCAL) alone. The horizontal separation between the two halves of both LCAL calorimeters was increased by $5 \mathrm{~mm}$ to reduce shadowing effects from SiCAL when it is in place.

2. SiCAL period: luminosity measurements after the September $1992 \mathrm{st}$

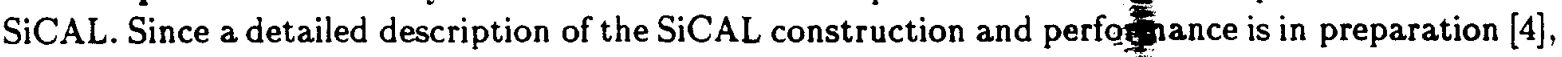
only aspects relevant to the cross section measurements are included here.

The analysis presented here is based upon a sample of 780000 charged fermion pairs collected by ALEPH at LEP in 1992, representing an integrated luminosity of $13.7 \mathrm{pb}^{-1}$ in the LCAL period $(\sqrt{s}=$ $(91.276 \pm 0.018) \mathrm{GeV})$ and $8.7 \mathrm{pb}^{-1}$ in the SiCAL period $(\sqrt{s}=(91.270 \pm 0.018) \mathrm{GeV})$. The LEP energies are given by the LEP energy group [5]. The fit results presented here are based upon 1300000 visible $\mathrm{Z}$ decays to fermion pairs accumulated by ALEPH over the past four years of data collection.

\section{Luminosity Determination}

\subsection{LCAL Luminosity Measurement}

The luminosity determination with the LCAL, covering the polar angular re a from 46 to $122 \mathrm{mrad}$, has been described in detail elsewhere [6]. The evaluation of the systematic error for 1992 proceeded in the same way as in the previous years. To check the accuracy of the understanding of the acceptance, the results using two different fiducial-side selections [6] were compared. The difference using all LCAL data from 1989 to 1992 is compatible with a systematic error of $0.21 \%$, whose determination with this method has fluctuated statistically from year to year. The total LCAL experimental systematic errors for this and the previous years become: $0.49 \%(1989-1990), 0.38 \%(1991)$ and $0.37 \%$ (1992 LCAL period) and are used in the electroweak fits.

\subsection{SiCAL Luminosity}

The SiCAL is a silicon-tungsten sampling electromagnetic calorimeter, 23 radiation lengths $\left(\mathrm{X}_{0}\right)$ in depth. It consists of two homogeneous cylindrical detectors surrounding the beam pipe at roughly $\pm 250 \mathrm{~cm}$ from the interaction point. The calorimeters cover the polar angular range from 24 to $58 \mathrm{mrad}$. Twelve layers of tungsten alternate with layers of silicon pad detectors. Each layer is segmented into 32 sectors in azimuth, each with 16 radial pad rows $5.225 \mathrm{~mm}$ wide. A total of 12288 pads are readout individually, allowing a full reconstruction of the transverse and longitudinal shower development. The internal alignment of the silicon detector elements and the measurement of the separation between half modules with a lightemitting diode optical system ensure a mechanical precision of the radial fiducial boundary of $18 \mu \mathrm{m}$. 
The analysis of the 1992 SiCAL luminosity is largely based upon the previous LCAL analysis [6], simplified by the homogeneity of the new detector. Bhabha scatterings are identified by requiring at least one reconstructed cluster in each endcap, side $A$ and side $B$, with an energy $\geq 20 \mathrm{GeV}$. The sum of their energies must be $\geq 55 \mathrm{GeV}$. The acoplanarity of these clusters is restricted to $150^{\circ} \leq \Delta \phi \leq 210^{\circ}$ in order to remove background from accidental coincidences of off-momentum beam particles. Radial cuts define the Bhabha acceptance. Energy sharing between pads at the fiducial boundary, summed over two layers in depth at $6 X_{0}$ and $8 X_{0}$, is used to determine the acceptance near the boundary. A "tight" fiducial selection on one side and a "loose" fiducial selection on the opposite side, alternated event by event, reduce the sensitivity of the selection to beam offsets. The tight side is sufficiently constrained to ensure full containment of showers from Bhabha events (radial pad rows 4 to 12 , inclusively), whereas the loose side permits clusters to lie within pad rows 2 to 15 , inclusively.

There is very good agreement between the data and the Monte Carlo simulation used to integrate the accepted low angle Bhabha cross section. Figure 1 shows the radial distribution for tight-side selected clusters, and Figure 2 shows the azimuthal distributions for events accepted with either side's tight radial selections. The periodic variation in $\phi$ is due to the offset of the beam with respect to the detector axis; it is well reproduced by the simulation.

The background to the luminosity selection comes almost entirely from two sources: 1) accidental coincidences of off-momentum beam particles, and 2) $t$-channel production of two or more hard photons, indistinguishable from a Bhabha scattering in this purely calorimetric monitor. The off-momentum background is evaluated using the SiCAL single-arm triggers and is found to be $8.5 \cdot 10^{-4}$ of the Bhabha rate, and is subtracted from the data. The $t$-channel hard photon background is calculated and used to correct the accepted Bhabha cross section; it corresponds to a $0.015 \%$ contamination of the selection. Other physics processes produce a background below $0.010 \%$.

Two cross-checks were used to verify the understanding of the Bhabha acceptance. First, the radius of the tight fiducial boundary was decreased by one padwidth; the ratio of accepted events to the Bhabha selection gives $1.237 \pm 0.001$ for data in good agreement with $1.238 \pm 0.001$ for the Monte Carlo simulation. Second, two independent azimuthal selections, sensitive to systematics from background subtraction or half-module separations, were compared: "horizontal" events were required to be in the horizontal plane $\pm 33.75^{\circ}$ and "vertical" events were required to be in the vertical plane $\pm 33.75^{\circ}$. The ratio of horizontally to vertically selected Bhabhas gives $1.003 \pm 0.003$ for data, in agreement with $1.006 \pm 0.003$ found for the Monte Carlo.

The experimental systematic errors are summarized in Table 1. The mechanical precision combined with the uncertainty on the $z$-distance between the calorimeters gives an ultimate luminosity precision of $0.068 \%$. Although more than two million Bhabha events were processed through the ALEPH simulation and reconstruction package, the 1992 estimated experimental precision is limited by the Monte Carlo statistics: $0.15 \%=0.09 \%(e x p.) \oplus 0.12 \%$ (mc stat. $)$. This is still reasonable given the statistical precision of the $\mathrm{Z}$ sample. The additional sources of experimental systematics come primarily from effects such as pad-to-pad energy precision and simulation of showers within the detector.

\subsection{LCAL-SiCAL Relative Luminosity Measurements}

Two comparisons of the LCAL and SiCAL luminosity measurements were performed to cross-check the LCAL measurements. First, indirectly, a fit to the hadronic cross section in the LCAL periods (1989 to September 1992) is compared to the hadronic cross section in the SiCAL period, the former corrected for the difference in $\sqrt{s}$. The ratio of the hadron cross section measured with SiCAL to the LCAL (energy corrected) cross section is $0.9981 \pm 0.0046$, where the statistical and systematic errors are combined, excluding the luminosity theoretical error discussed below. 


\section{ALEPH}

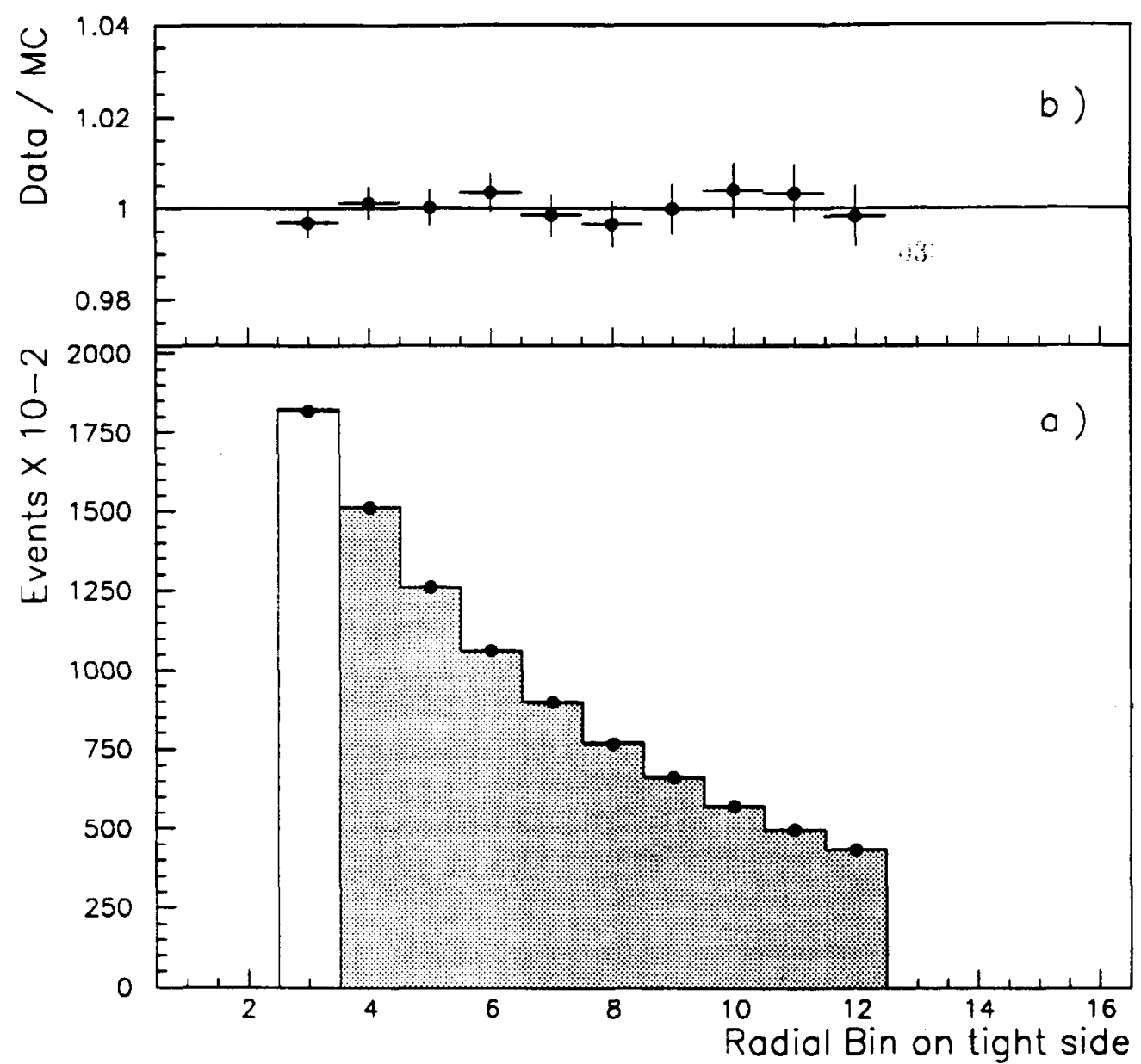

Figure 1: a) Radial distribution for the tight-side selection (final selection shown as shaded zone). Data are plotted as points; Monte Carlo shown as a histogram.

b) Ratio of data to Monte Carlo. 


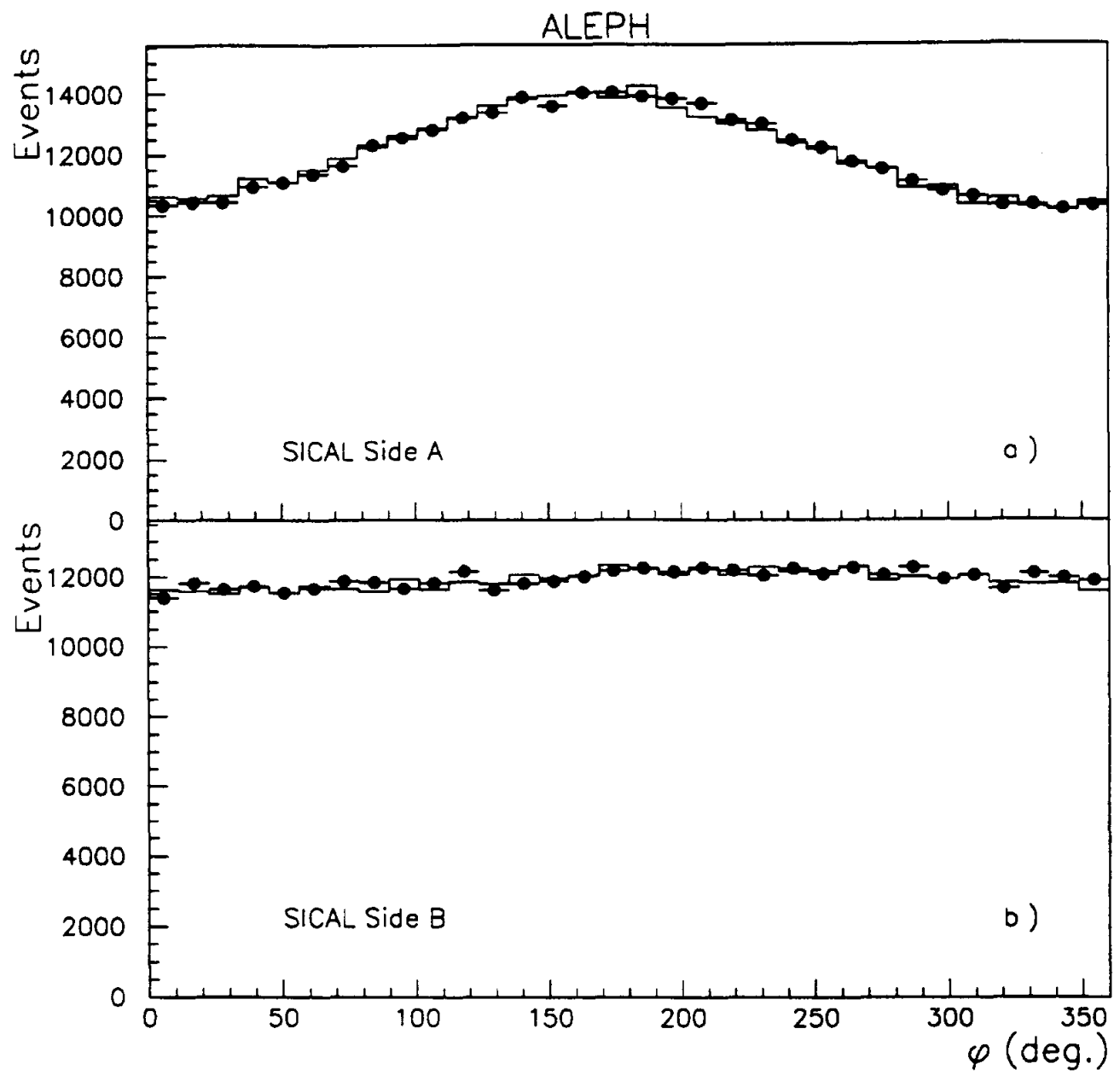

Figure 2: a) $\phi$ distribution for side A tight selection.

b) $\phi$ distribution for side $B$ tight selection, for accepted Bhabha events. The data are plotted as points and the Monte Carlo is shown as a histogram. The $\phi$ variation is due to different offsets of the beam with respect to the axes of the side $\mathrm{A}$ and side $\mathrm{B}$ detectors. 
Background estimation:

- Off-momentum beam particles $\quad 0.018 \%$

- Physics sources $\quad 0.010 \%$

Trigger efficiency $\quad 0.010 \%$

Radial fiducial cuts:

- mechanical precision and $\mathrm{z}$ position $\quad 0.068 \%$

- beam-module relative tilt and alignments $0.035 \%$

- energy sharing cuts $\quad 0.044 \%$

- shower parametrization and simulation $\quad 0.023 \%$

Energy cuts $\quad 0.015 \%$

Acoplanarity cut $\quad 0.005 \%$

Simulation statistics $\quad \therefore \quad 0.120 \%$

TOTAL experimental uncertainty $\quad 0.153 \%$

Table 1: Summary of SiCAL luminosity systematic errors.

A second, direct test was made comparing the LCAL measured luminosity during the SiCAL period. In contrast to previous years, the SiCAL shadow below $59 \mathrm{mrad}$ on the LCAL detector could slightly disturb the latter's precision. The ratio between the LCAL and SiCAL integrated luminosities in the SiCAL period is $0.9988 \pm 0.0039$ (stat) \pm 0.0039 (syst), where the statistical error includes both data and Monte Carlo simulation statistics and the systematic error excludes the theoretical uncertainty. The shadowing effect is included and is estimated to contribute an uncertainty of $\leq 0.1 \%$.

Combining the two tests, and interpreting the ratio of hadron cross sections as a luminosity ratio, gives an LCAL to SiCAL luminosity measurement ratio of $0.9984 \pm 0.0027$ (stat) \pm 0.0034 (syst).

\subsection{Luminosity Theoretical Error}

The Bhabha reference cross sections for LCAL and SiCAL acceptances during both periods have been calculated using the Monte Carlo program BHLUMI [7]. BHLUMI is a multi-photon $\mathcal{O}(\alpha)$ event generator with exclusive exponentiation. The full $\mathcal{O}(\alpha)$ Z-exchange correction has been done with the BABAMC generator [8]. The electroweak correction is four times smaller for SiCAL with respect to LCAL due to the former's acceptance at lower angles. This reflects in a smaller theoretical uncertainty for the SiCAL Bhabha cross section [9]: the theoretical systematic error for LCAL is estimated to be $0.28 \%$ (as is 1991 [1]) and $0.25 \%$ for SiCAL. The separate contributions are summarized in Table 2.

\section{Measurements of Cross Sections: Id Forward-Backward Asymmetries}

The selections for hadronic and leptonic events, except the $\tau^{+} \tau^{-}$selection, are not changed from those in $[1,2]$. The evaluations of the selection systematics for the hadrons, Bhabhas and muon-pairs are 

(1) $\mathcal{O}\left(\alpha^{2}\right)$ LL BHLUMI
$0.15 \% \quad 0.15 \%$
(2) $\mathcal{O}\left(\alpha^{2}\right)$ SL BHLUMI
$0.09 \% \quad 0.09 \%$
(3) Z-exchange $\mathcal{O}(\alpha)$ BABAMC
$0.03 \% \quad 0.03 \%$
(4) Z-exchange $\mathcal{O}\left(\alpha^{2}\right)$ LL missing in BABAMC
$0.06 \% \quad 0.015 \%$
(5) Z-exchange $\mathcal{O}\left(\alpha^{2}\right)$ SL missing in BABAMC
$0.06 \% \quad 0.015 \%$
(6) Vacuum polarization
$0.08 \% \quad 0.05 \%$

Total theoretical uncertainty

$0.28 \% \quad 0.25 \%$

Table 2: Summary of theoretical systematic errors from [9] for the two luminosity reference cross sections. QED leading-log (LL) and sub-leading (SL) uncertainties ( 1 and 2 ) and the Z-exchange correction uncertainties (4 and 5) are each added linearly.

discussed below. The improved $\tau^{+} \tau^{-}$selection and its corresponding systematic error are presented in detail. The flavor-blind lepton selection, called "common lepton" hereafter, has not been changed from $[1,2]$.

The measured cross sections for the hadronic (selections averaged) and lepton-pair final states are shown in Table 3 . The lepton forward-backward asymmetries $A_{F B}$, presented in Table 4 are determined by a maximum likelihood fit of the function

$$
\frac{d \sigma}{d \cos \theta^{*}}=C \cdot\left(1+\cos ^{2} \theta^{*}+\frac{8}{3} A_{F B} \cos \theta^{*}\right) \cdot F\left(\cos \theta^{*}\right)
$$

to the $\cos \theta^{*}$ angular distributions. The scattering angle, $\theta^{*}$, is defined as

$$
\cos \theta^{*}=\cos \frac{1}{2}\left(\theta_{1}+\pi-\theta_{2}\right) / \cos \frac{1}{2}\left(\theta_{1}-\pi+\theta_{2}\right),
$$

where $\theta_{1}$ and $\theta_{2}$ are the polar angles of the vector sum of the track momenta in the hemispheres corresponding to the outgoing lepton and antilepton [2]. $F\left(\cos \theta^{*}\right)$ describes the effect of the $t$-channel exchange for the $e^{+} e^{-}$channel and $C$ is a normalization constant.

\subsection{Hadronic Selection Systematics}

Two hadron selection methods are used, one based on the energy and multiplicity of charged particles in the Time Projection Chamber (TPC) and the other based on the total calorimetric energy. The efficiencies are $97.47 \%$ and $99.07 \%$ respectively, where the loss in efficiency for the charged-track selection comes essentially from low-angle events which fail either the multiplicity or the energy cuts. The cross sections are given in Table 3 for the individual selections and for their average. The event selection cuts out events with large initial state radiation. The cross sections are given corrected up to $\sqrt{s}>0.1 \sqrt{s}$, where $\sqrt{s}$ is the mass of the $e^{+} e^{-}$pair after initial state radiation.

Increased data and higher Monte Carlo statistics have improved the understanding of the tau pair 


\begin{tabular}{lcc}
\hline & LCAL period & SiCAL period \\
Measurement & & \\
\hline & & \\
$\mathcal{L}_{\text {int }}\left(\mathrm{nb}^{-1}\right)$ & $13684.0 \pm 23.4 \pm 43.8$ & $8749.0 \pm 10.2 \pm 13.1$ \\
& $\pm 38.3(\mathrm{th})$. & \pm 21.9 (th.) \\
Number of selected $q \bar{q}$ & & \\
$e e$ & 16647 & 264610 \\
$\mu \mu$ & 17082 & 10725 \\
$\tau \tau$ & 16274 & 10879 \\
common leptons & 58566 & 10385 \\
& & 37607 \\
$\sigma_{\text {had }}(\mathrm{nb}) \quad$ Charged tracks: & $30.719 \pm 0.071 \pm 0.141$ & $30.575 \pm 0.070 \pm 0.103$ \\
$\sigma_{\text {had }}(\mathrm{nb})$ Calorimeter: & $30.710 \pm 0.071 \pm 0.139$ & $30.566 \pm 0.069 \pm 0.102$ \\
$\sigma_{\text {had }}(\mathrm{nb}) \quad$ Average: & $30.715 \pm 0.071 \pm 0.137$ & $30.570 \pm 0.069 \pm 0.099$ \\
& & \\
$\sigma_{\text {ee }}(\mathrm{nb})$ & $1.506 \pm 0.014 \pm 0.009$ & $1.519 \pm 0.017 \pm 0.007$ \\
$\sigma_{\mu \mu}(\mathrm{nb})$ & $1.499 \pm 0.012 \pm 0.009$ & $1.484 \pm 0.014 \pm 0.008$ \\
$\sigma_{\tau \tau}(\mathrm{nb})$ & $1.497 \pm 0.012 \pm 0.008$ & $1.494 \pm 0.015 \pm 0.007$ \\
$\sigma_{\text {common leptons }}(\mathrm{nb})$ & $4.503 \pm 0.024 \pm 0.026$ & $4.525 \pm 0.027 \pm 0.022$ \\
\hline
\end{tabular}

Table 3: Integrated luminosities, event numbers and hadron and lepton cross sections with statistical and systematic errors for 1992. The data are separated for running before SiCAL installation, LCAL period $(91.276 \mathrm{GeV})$, and afterwards, SiCAL period $(91.270 \mathrm{GeV})$. The theoretical error (th.) is shown explicitly for the luminosities. The luminosity errors (theoretical error included) are added in quadrature in the cross sections shown here.

background in the calorimetric selection. The correction is $(0.46 \pm 0.06) \%$, where the uncertainty is reduced by more than a factor two with respect to the previous publications $[1,2]$.

The systematic error of the charged track based selection has been re-evaluated to take advantage of the increased statistics. The backgrounds are small, $(0.32 \pm 0.03) \%$ coming from $\tau^{+} \tau^{-}$events and $(0.25 \pm 0.08) \%$ from two-photon interactions. The limited precision in the simulation of the detector response for the cut variables [2] is responsible for a systematic error of $0.4 \cdot 10^{-3}$.

The uncertainty in the extrapolation to full acceptance coming from the hadronization model has been estimated using real events in which the thrust axis is nearly normal to the beam direction $\left(\left|\cos \theta_{\text {Thrust }}\right|<\right.$ 0.2 ). The events were rotated randomly in space and tracks falling outside the detector acceptance were eliminated. The acceptance was then recalculated by weighting the events passing the hadron selection with the proper thrust angular distribution. This procedure was repeated with fully simulated events. A difference in the efficiency of $(1.1 \pm 0.4) \cdot 10^{-3}$ was found. The selected events are well contained in the detector, the loss in average track multiplicity being $<1 \%$; the probability that they are not identified as hadronic events is negligible. However, two classes of hadronic decays are not represented in the sample: low charged multiplicity events and events with strong initial state bremsstrahlung. Although the second class is well simulated in the Monte Carlo, the uncertainty in the fraction of events of the first class is large. The estimated uncertainty due to these events is $0.8 \cdot 10^{-3}$, giving a total uncertainty in the efficiency of $1.4 \cdot 10^{-3}$. Combining all the errors assigned to detector response, Monte Carlo, background 
Measurement $\mathrm{LCAL}+\mathrm{SiCAL}$ periods

$\begin{array}{ll}A_{F B}^{e e} & 0.0115 \pm 0.0074 \pm 0.0029 \\ A_{F B}^{\mu \mu} & 0.0101 \pm 0.0059 \pm 0.0010 \\ A_{F B}^{r r} & 0.0052 \pm 0.0067 \pm 0.0005\end{array}$

Table 4: Forward-backward asymmetries for lepton pairs. The data are presented for the combined LCAL and SiCAL periods, where the average energy is $91.274 \mathrm{GeV}$. Both the statistical and systematic errors are given.

and modeling gives a total systematic error for the track-based selection of $1.7 \cdot 10^{-3}$.

The systematic errors for both selections are summarized in Table 5 . Since they use different detector components for the identification, the two selections are considered independent. Therefore the systematic error of the average is obtained by combining the systematic errors of the two selection efficiencies, assuming they are uncorrelated, with the other uncertainties (Monte Carlo statistics and background), assuming they are fully correlated between both selections. The overall systematic uncertainty in the combined cross section is then $0.14 \%$.

\begin{tabular}{lcc}
\hline & \multicolumn{2}{c}{ Hadronic selection } \\
Source & Charged track & Calorimeter \\
& & \\
\hline & 0.4 & 1.1 \\
detector response & 1.4 & negl. \\
modeling & 0.4 & 0.4 \\
Monte Carlo stat. & & 0.8 \\
background: & 0.8 & 0.3 \\
$\gamma \gamma$ & negl. & 0.6 \\
$e^{+} e^{-}$ & 0.3 & \\
$\tau^{+} \tau^{-}$ & & 1.6 \\
\hline Total & 1.7 & \\
\hline
\end{tabular}

Table 5: Systematic errors $\left(10^{-3}\right)$ for hadronic selections.

\section{2 $\mathrm{Z} \rightarrow e^{+} e^{-}$Selection Systematics}

The selection of electron pairs is based on the sum of the momenta of the two tracks with highest momenta and the sum of the energy in the electromagnetic calorimeter and in the regions of the hadron calorimeter along the cracks of the electromagnetic one. The selection efficiency and the $\tau$ background 
systematic errors have been re-evaluated since [1]; the error on the global efficiency and $\tau$ background together is $0.3 \%$. The uncertainty in the extreme backward bin of $\cos \theta^{*},[-0.9,-0.8]$, was re-evaluated by moving the most sensitive cut, the one on the sum of momenta and calorimetric energy [2], by $\pm 10 \%$. Agreement between data and Monte Carlo is consistent with a $1 \%$ uncertainty for this bin. The resulting total systematic error for the cross section is $0.41 \%$, and the error for the $A_{F B}$ measurement is $0.29 \%$. The theoretical uncertainty in the $t$-channel subtraction due to inherent limitations of the calculation in the ALIBABA program [10] contributes $0.23 \%$ and $0.25 \%$ to these errors, respectively.

The number of selected events (before corrections, $t$-channel subtraction, etc.), measured cross section and forward-backward asymmetry are given in Tables 3 and 4 .

\subsection{Z $\rightarrow \mu^{+} \mu^{-}$Selection Systematics}

The selection of muon-pair events is based on their penetrating power, which leads to signals in the last planes of the hadron calorimeter and in the two layers of muon chambers that surround ALEPH [2]. The systematic error for the $\mu$-pair cross section is unchanged from [1], $0.5 \%$. Two studies have been used to set an upper limit on the systematic error for the measurement of the forward-backward asymmetry. First, a comparison has been made between the standard selection and the one based on minimum ionizing signals in ECAL and HCAL [2], both of which have different background components. An uncertainty of $7 \cdot 10^{-4}$ on $A_{F B}$ is inferred from the difference between both selections. Second, the accuracy of the tracking in the very forward region has been studied by enlarging the selection to accept tracks with $|\cos \theta|<0.98$ and events with $\left|\cos \theta^{*}\right|<0.95$. An upper limit of $6 \cdot 10^{-4}$ is obtained for the systematic error from this source. Adding the uncertainties from both sources in quadrature gives a total systematic uncertainty on $A_{F B}$ of $0.10 \%$.

The number of selected events, and values of the measured cross section and forward-backward asymmetry are shown in Tables 3 and 4 .

\subsection{Improved $\mathrm{Z} \rightarrow \tau^{+} \tau^{-}$Selection and Systematics}

\subsection{1 $\tau^{+} \tau^{-}$Selection and Cross Section}

The new selection of $\tau$-pair events starts by dividing the event in two hemispheres by a plane perpendicular to the thrust axis. Two "jets" are defined by clustering together all charged and neutral objects present in each hemisphere, which are found using the energy flow algorithm described in [11]. The $\tau$ direction is given by the vectorial sum of the momenta of all the objects in the jet. The most energetic charged track in each hemisphere is called leading track.

The event is required to have at least 2 and at most 8 charged tracks in the polar angular range $|\cos \theta|<0.95$ and at least one charged track in each hemisphere. The scattering angle $\theta^{*}$, defined using the charge and the direction of jets, has to satisfy the condition $\left|\cos \theta^{*}\right|<0.9$. In order to reduce the $\gamma \gamma$ background the event must have an acollinearity, defined as $180^{\circ}$ minus the angle between the two jets, smaller than $20^{\circ}$, and either a ratio of total reconstructed energy, $E_{\text {rec }}$, to beam energy, $E_{\text {beam }}$, $E_{\text {rec }} / E_{\text {beam }}>0.350$ or an absolute difference between the transverse momenta of the jets greater than $3 \mathrm{GeV} / c$. Cosmic ray reduction is obtained by asking for at least one charged track to originate from within a cylinder of length $10 \mathrm{~cm}$ and radius $2 \mathrm{~cm}$ coaxial with the beam and centered on the measured collision point. If both hemispheres have either more than one charged track or an invariant mass greater than $1 \mathrm{GeV} / c^{2}$, further cuts are applied to reject hadronic events. Defining $n_{o b, i}$ and $\theta_{i}^{o p}$ as the number of charged and neutral objects and the maximum angle between two charged tracks in the hemisphere $i$ respectively, the requirements are $n_{o b, 1} \cdot n_{o b, 2}<75$ and $\theta_{1}^{o p}+\theta_{2}^{o p}<0.25 \mathrm{rad}$. 
Bhabha and dimuon events are rejected by requiring the sum of the energy of the two leading charged tracks to be less than 1.6 $E_{\text {beam }}$. The fractions of Bhabha and dimuon events remaining at this level are $17 \%$ and $2.5 \%$ respectively. The remaining events are then tagged using the lepton identification described in [11] applied with very loose cuts to keep a high efficiency. In addition, charged tracks less than $3 \mathrm{~cm}$ away from ECAL cracks are called electrons if they are not identified as muons. Bhabhalike events, i.e. events where all the charged tracks are identified as electrons, are rejected using two variables. First, the total energy is found as $E_{\text {tot }}=E_{\text {rec }}+E_{\text {rad }}$, where $E_{\text {rad }}$ is the energy of a radiated photon collinear to the beam, computed using the jet directions and imposing 4-momentum conservation. Second, in order to tag possible losses of photons due to ECAL cracks, a variable $\left(D_{g, i}\right)$ is defined by taking the tangent to the leading track in each hemisphere at the origin, extrapolating it to ECAL, and computing its minimum distance from a crack. Bhabha rejection is then obtained by applying a staggered cut in the variables $D_{\min }=\min \left(D_{g, 1}, D_{g, 2}\right)$ and $E_{t o t}$. The $\tau$-pair events must have $E_{t o t} / E_{\text {beam }}<1.6$ when $D_{\min }>6 \mathrm{~cm}$, or $E_{\text {tot }} / E_{\text {beam }}<1.4$ when $D_{\min }<6 \mathrm{~cm}$ (see fig. $3 \mathrm{a}$ and b). Dimuon-like events are defined either if both leading tracks are identified as muons, or if one leading track is identified as a muon and the opposite hemisphere has a momentum in excess of $0.9 \cdot E_{\text {beam }}$. Dimuon rejection is obtained by requiring $E_{\text {tot }} / E_{\text {beam }}<1.8$. Figure $3 \mathrm{c}$ ) shows the $E_{\text {tot }}$ distribution for data and simulated dimuon events.

After this selection 26659 events remain. The overall acceptance is $78.12 \pm 0.23 \%$, arising from an angular acceptance of $85.7 \%$ and a selection efficiency inside the acceptance of $91.16 \pm 0.27 \%$. The error has been obtained by combining the uncertainties from the selection criteria, evaluated either by comparing data with Monte Carlo or by using artificial tau pairs formed from the data with tau's from different events [2]. The most important uncertainty comes from hadron rejection cuts due to the limited statistics of such artificial tau pairs. Backgrounds from electron pairs, muon pairs, and cosmic rays have been estimated using data to be respectively $(0.66 \pm 0.14) \%,(0.25 \pm 0.05) \%$, and $(0.17 \pm 0.03) \%$. Monte Carlo simulations of $\gamma \gamma$ and hadronic events have been used to estimate the contamination to be $(0.31 \pm 0.04) \%$ and $(0.25 \pm 0.03) \%$ respectively. The overall background subtraction amounts to $(1.64 \pm 0.16) \%$. The number of selected events and the cross section are given in Table 3 for LCAL and SiCAL periods. The systematic error is the sum in quadrature of background, efficiency and luminosity contributions.

\subsection{2 $\tau^{+} \tau^{-}$Forward-Backward Asymmetry}

The measurement of the forward-backward asymmetry, $A_{F B}^{r \tau}$, requires additional cuts in order to minimize the bias from the remaining Bhabha background. To account for possible effects from a non-uniform efficiency with $\cos \theta^{*}, A_{F B}$ has been measured differentially in five bins of $\cos \theta^{*}$, the final result being the weighted mean of the five values. Events are required to have one or three charged tracks per hemisphere and zero total charge. In the one prong-one prong (1-1) subsample Bhabha events are reduced to a negligible level either, 1) if one track is identified as an electron, by asking $E_{\text {tot }} / E_{\text {beam }}<1.7$ when $\left|\cos \theta^{\circ}\right|<0.72$, and $<1.4$ otherwise, or 2$)$ if both tracks are identified as electrons, by requiring the energy ratio to be smaller than 1.2 and 0.6 , respectively.

After these additional requirements the number of selected events is 22063 . The Bhabha contamination is determined from the data to be smaller than $2.5 \cdot 10^{-3}$, giving rise to a $4.5 \cdot 10^{-4}$ systematic error on $A_{F B}$. The residual symmetrical contamination amounts to $(0.40 \pm 0.04) \%$, giving a contribution to the uncertainty in $A_{F B}$, of $\pm 0.6 \cdot 10^{-4}$. The probability to mis-measure the electric charge in a hemisphere, measured by studying the total charge distribution for 1-1 and 1-3 events, is found to be smaller than $10^{-2}$, giving a correction to $A_{F B}$ below $10^{-4}$. The effect of track-insensitive regions in the Time Projection Chamber is negligible because of the $\left|\cos \theta^{*}\right|$ cut at 0.9 . The same effect in the calorimeters is investigated by comparing hemispheres which are selected only based on charged tracks with hemispheres which include calorimeter energy. The estimate of the systematic error due to this effect is $2.5 \cdot 10^{-4}$. The $A_{F B}^{\tau \tau}$ value for the combined LCAL and SiCAL periods is given in Table 4. 

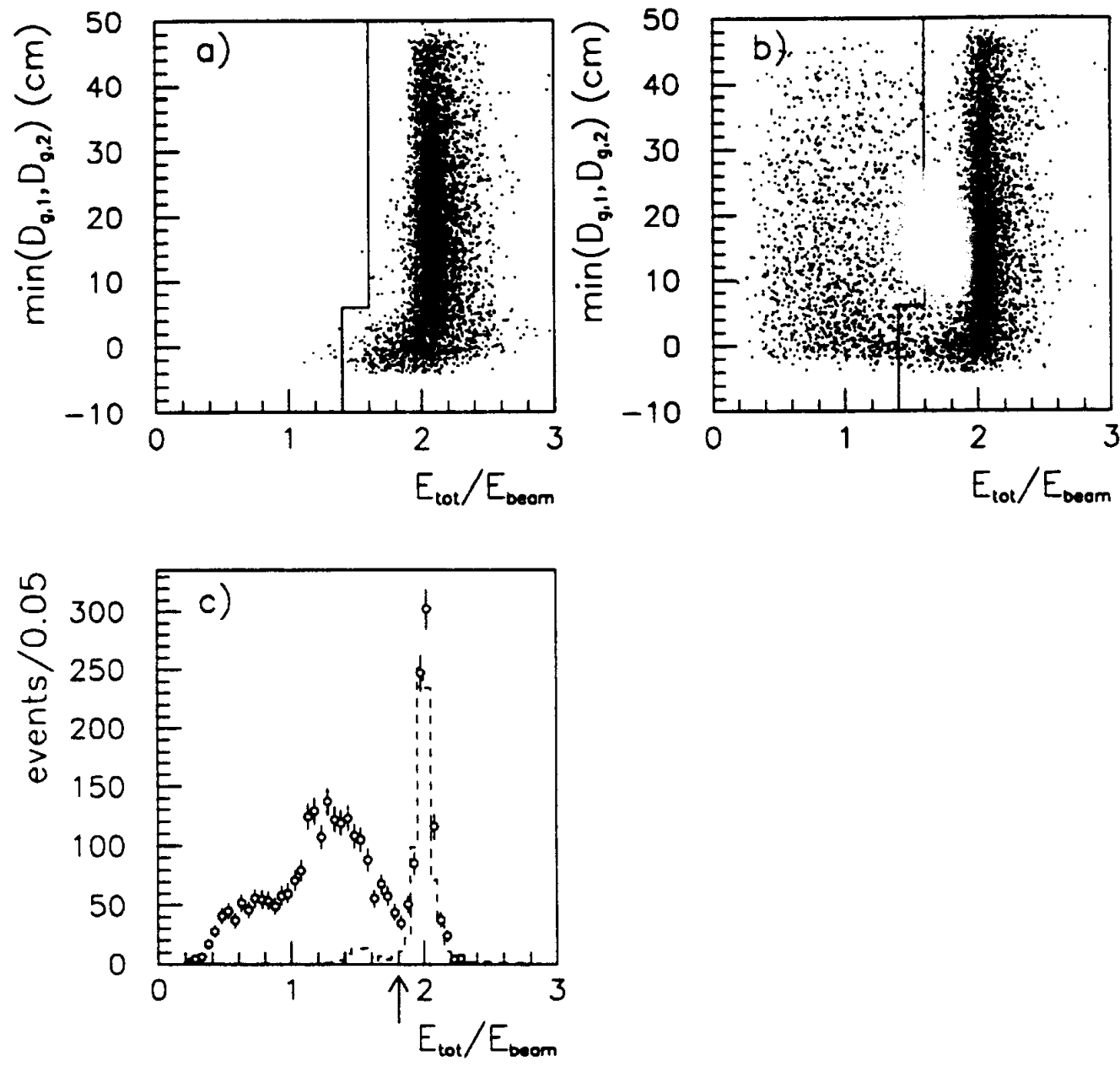

Figure 3: a) Minimum distance of the leading tracks to an ECAL crack versus $E_{\text {tot }}$ for Bhabha Monte Carlo events; b) the same distribution for Bhabha-like events in the data; c) $E_{\text {tot }}$ distribution for data satisfying dimuon-like criteria (open circles) and dimuon Monte Carlo events (dashed histogram). See text for definitions. 
The determination of $A_{F B}$ was repeated using the selection previously published [1,2], based mainly on identifying muons and pions using information from the calorimeters. In addition the Bhabha background has been reduced by $75 \%$ with respect to [1] with a combined cut on track momentum and energy in the electromagnetic calorimeter, $E_{\text {cal }}^{2}+P^{2}$. The selection efficiency is $84 \%$ within $|\cos \theta|<0.9$. The result is $A_{F B}^{\tau r}=0.0048 \pm 0.0065$. The systematic error of this analysis was evaluated and found to be 0.0005 , essentially from the same sources as in the analysis above, and also applies to the previously published $A_{F B}^{T r}$ data $[1,2]$. The forward-backward asymmetries from the two selection procedures agree and have negligible systematic errors. Since the new selection is also used for the determination of the tau-pair cross section, its value for the forward-backward asymmetry is given in Table 4 .

\subsection{Common Lepton Cross Section}

The flavour-blind lepton-pair selection is based on the measurement of charged particles and does not attempt to identify the individual lepton species. The selection has not been changed from [1,2]. The resulting cross section is given in Table 3 . Its systematic error remains the same, $0.4 \%$, and is essentially independent to the systematic errors of the individual lepton analyses, which come mainly from particle identification. As a cross-check of the systematic errors of the individual lepton analyses, the cross-section obtained with the common lepton selection has been compared with the sum for the three individual lepton channels. The difference for the two periods combined is

$$
\sigma_{\text {common leptons }}-\left(\sigma_{e e}+\sigma_{\mu \mu}+\sigma_{\tau \tau}\right)=(0.012 \pm 0.007) \mathrm{nb}
$$

where the error includes only the non-common statistical uncertainty.

\section{Fits to Cross Sections and Forward-Backward Asymme- tries}

The 1992 hadron and individual lepton cross sections and forward-backward asymmetries were fit along with the accumulated 1989, 1990 and 1991 ALEPH data using the MIZA [12] lineshape fitting program as in [1] updated with the results in [13]. A 5-parameter fit assuming lepton universality and a 9-parameter fit without lepton universality are made in the same way as in [1]. The results are shown in Table 6. In the following $R_{l}$ is defined as the ratio of the $\mathrm{Z}$ hadronic partial width to the leptonic partial width, $\Gamma_{l l}$, for massless final-state leptons (obtained from $R_{e}, R_{\mu}$ and $R_{\tau}$ correcting for mass effects). The 1992 data taken at the $Z$ peak do not contribute to the values of $M_{Z}$ and $\Gamma_{Z}$, both of which remain unchanged from [1]. The errors in the peak forward-backward asymmetries, $A_{F B}^{0, i}$, include a 0.0010 component from the uncertainty in the LEP center of mass energies.

The fit parameters can be used to derive the leptonic and hadronic partial widths, $\Gamma_{11}$ and $\Gamma_{\text {had }}$, respectively, their branching ratios and the invisible width, $\Gamma_{i n v}=\Gamma_{Z}-\Gamma_{h a d}-\left(\Gamma_{e e}+\Gamma_{\mu \mu}+\Gamma_{\tau \tau}\right)$. The results are shown in Table 7. Using the electroweak Standard Model value for $\Gamma_{\nu} / \Gamma_{u}(1.992 \pm 0.003)$, the ratio of the invisible width to the leptonic one, assuming $\Gamma_{i n v}=N_{\nu} \Gamma_{\nu}$, gives the number of light neutrino species

$$
N_{\nu}=2.983 \pm 0.034 \text {. }
$$

Alternatively, assuming three neutrino species allows one to determine the ratio $\Gamma_{\nu} / \Gamma_{u}=1.981 \pm 0.022$.

Both $\sigma_{h}^{0}$ and $R_{i}$, within the context of the minimal Standard Model, depend weakly on the masses of the top quark and Higgs boson, making them ideal parameters to test for departures from the standard electroweak theory. The principal remaining uncertainty comes from the dependence of $R_{l}$ and to a more 


\section{Parameter No lepton univ. Lepton univ.}

\begin{tabular}{lcc}
\hline & & \\
$\mathrm{M}_{Z}(\mathrm{GeV})$ & $91.187 \pm 0.009$ & $91.187 \pm 0.009$ \\
$\Gamma_{Z}(\mathrm{GeV})$ & $2.501 \pm 0.012$ & $2.501 \pm 0.012$ \\
$\sigma_{h}^{0}(\mathrm{nb})$ & $41.60 \pm 0.16$ & $41.60 \pm 0.16$ \\
$\mathrm{R}_{e}$ & $20.59 \pm 0.15$ & - \\
$\mathrm{R}_{\mu}$ & $20.83 \pm 0.15$ & - \\
$\mathrm{R}_{\tau}$ & $20.70 \pm 0.16$ & - \\
$\mathrm{R}_{l}$ & - & $20.69 \pm 0.09$ \\
$A_{F B}^{0, e}$ & $0.0185 \pm 0.0066$ & - \\
$A_{F B}^{0, \mu}$ & $0.0146 \pm 0.0048$ & - \\
$A_{F B}^{0,0}$ & $0.0197 \pm 0.0056$ & - \\
$A_{F B}^{0, l}$ & - & $0.0171 \pm 0.33$ \\
$\chi^{2}$ & & \\
& & \\
\hline
\end{tabular}

Table 6: Fit results for cross sections and forward-backward asymmetries using all the ALEPH accumulated data $(1989,1990,1991$ [1] and 1992). The full correlation matrices for the results are given in Appendix A.

limited degree $\sigma_{h}^{0}$ on the strong coupling constant $\alpha_{s}$. Figure 4 shows the probability contours for the parameters $\mathrm{R}_{l}$ and $\sigma_{h}^{0}$ given by the fit, along with the Standard Model prediction for 3 light neutrino generations, using a third-order expansion in $\alpha_{n}$ in the $\overline{M S}$ scheme as in [1]. The value of $\alpha_{1}$ shown in Table 7 is obtained from $R_{l}$ using the formula

$$
R_{l}=R_{l}^{0}\left(1+1.05 \frac{\alpha_{s}}{\pi}+(0.9 \pm 0.1)\left(\frac{\alpha_{s}}{\pi}\right)^{2}-13\left(\frac{\alpha_{s}}{\pi}\right)^{3}\right)
$$

where $R_{l}^{0}=19.936 \pm 0.029$ in the Standard Model $^{2}$. The central value assumes top quark and Higgs masses of $150 \mathrm{GeV}$ and $300 \mathrm{GeV}$ respectively, and the error includes the effects of changing the top mass from $100 \mathrm{GeV}$ to $200 \mathrm{GeV}$ and the Higgs mass from $60 \mathrm{GeV}$ to $1 \mathrm{TeV}$.

The vector and axial vector couplings to fermions are determined from the forward-backward asymmetries and the leptonic partial widths. $A_{F B}^{0, l}$ is used to obtain the ratio of the vector and axial vector couplings

$$
g_{V}^{2}\left(M_{Z}^{2}\right) / g_{A}^{2}\left(M_{Z}^{2}\right)=0.0058 \pm 0.0011
$$

assuming lepton universality. This is interpreted as a measurement of the weak effective mixing angle, $\sin ^{2} \theta_{e f f}^{\text {lept }} \equiv \frac{1}{4}\left(1-g_{V}\left(M_{Z}^{2}\right) / g_{A}\left(M_{Z}^{2}\right)\right)$, according to the definition used in [18]:

$$
\sin ^{2} \theta_{e \rho \rho}^{\text {lept }}=0.2310 \pm 0.0019
$$

The 9-parameter fit is repeated using the additional constraint coming from the measurement of $\tau$ polarization [14] from the 1989-1991 ALEPH data sample, to give improved results for the effective vector couplings shown in Table 8. Using these results together with the previous ALEPH measurements of the

\footnotetext{
${ }^{2}$ The calculation has been done using the electroweak library of the MIZA program
} 


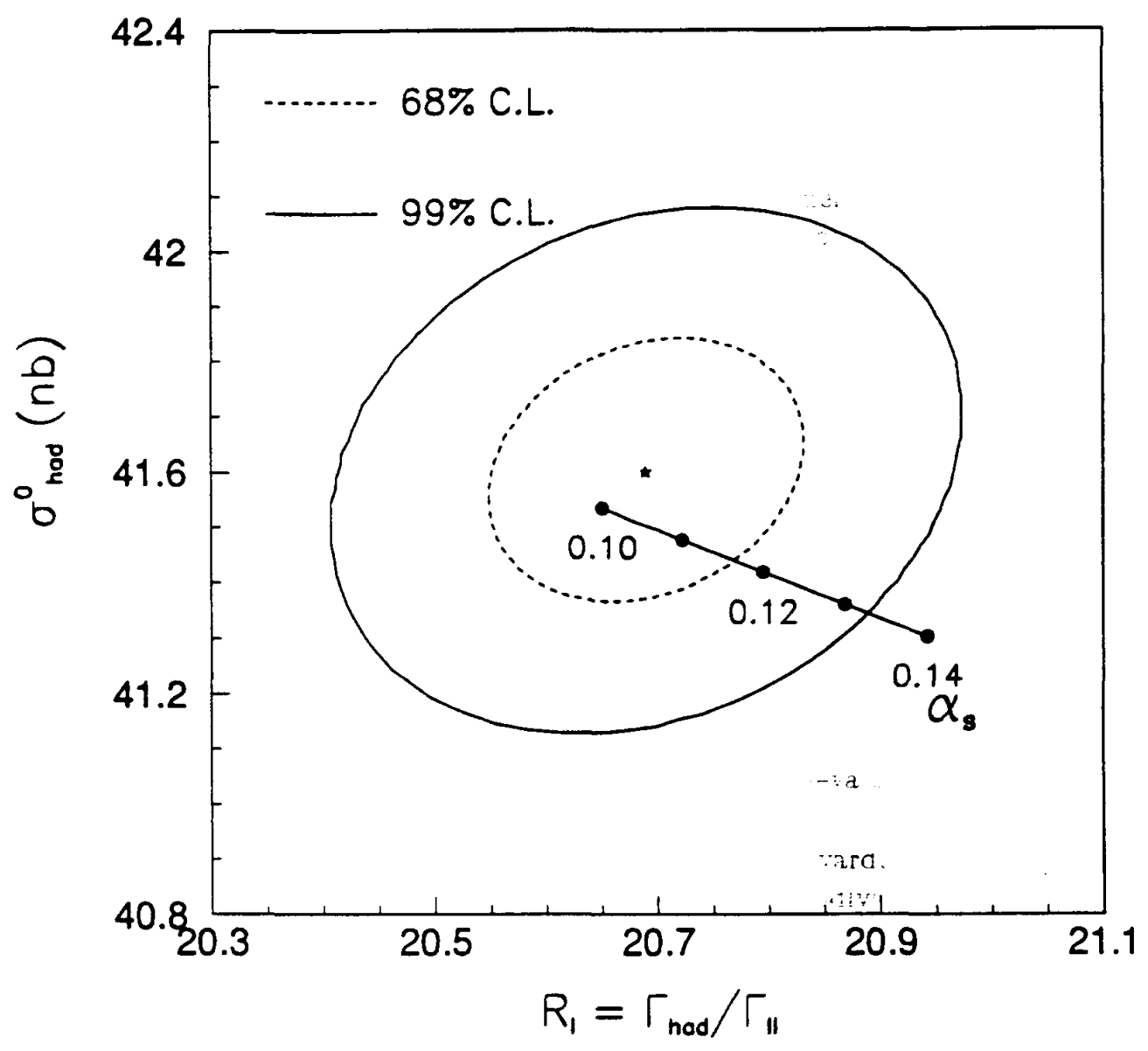

Figure 4: Contours of constant $\chi^{2}$ for $\sigma_{h}^{0}$, the hadronic peak cross section, as a function of $\mathrm{R}_{l}=\Gamma_{\text {had }} / \Gamma_{\| l}$ together with the Standard Model prediction as a function of the QCD coupling constant $\alpha_{s}\left(M_{Z}^{2}\right)$. 


\begin{tabular}{|c|c|c|}
\hline Derived parameter & No lepton univ. & Lepton univ. \\
\hline$\Gamma_{\text {had }}(\mathrm{MeV})$ & 一 & $1746 \pm 10$ \\
\hline$\Gamma_{l l}(\mathrm{MeV})$ & - & $84.40 \pm 0.43$ \\
\hline$\Gamma_{i n v}(\mathrm{MeV})$ & 一 & $501 \pm 6$ \\
\hline$\Gamma_{i n v} / \Gamma_{u}$ & - & $5.942 \pm 0.067$ \\
\hline$\Gamma_{e e}(\mathrm{MeV})$ & $84.61 \pm 0.49$ & - \\
\hline$\Gamma_{\mu \mu}(\mathrm{MeV})$ & $83.62 \pm 0.75$ & 一 \\
\hline$\Gamma_{\tau \tau}(\mathrm{MeV})$ & $84.18 \pm 0.79$ & - \\
\hline $\mathrm{Br}(\mathrm{Z} \rightarrow$ hadrons $)(\%)$ & $69.66 \pm 0.29$ & $69.83 \pm 0.23$ \\
\hline $\mathrm{Br}\left(\mathrm{Z} \rightarrow l^{+} l^{-}\right)(\%)$ & - & $3.375 \pm 0.009$ \\
\hline $\operatorname{Br}\left(\mathrm{Z} \rightarrow e^{+} e^{-}\right)(\%)$ & $3.383 \pm: \quad 3$ & - \\
\hline $\operatorname{Br}\left(Z \rightarrow \mu^{+} \mu^{-}\right)(\%)$ & $3.344 \pm: \quad 26$ & - \\
\hline $\operatorname{Br}\left(\mathrm{Z} \rightarrow \tau^{+} \tau^{-}\right)(\%)$ & $3.366 \pm 0.028$ & - \\
\hline$\sigma_{u l}^{0}(\mathrm{nb})$ & - & $2.011 \pm 0.010$ \\
\hline$\sigma_{e e}^{0}(\mathrm{nb})$ & $2.021 \pm 0.015$ & - \\
\hline$\sigma_{\mu \mu}^{0}(\mathrm{nb})$ & $1.997 \pm 0.015$ & - \\
\hline$\sigma_{\tau \tau}^{\mu \mu}(\mathrm{nb})$ & $2.010 \pm 0.016$ & - \\
\hline$\alpha_{s}$ & - & $0.108 \pm 0.012$ \\
\hline$g_{v 1}\left(M_{Z}^{2}\right)$ & - & $-0.038 \pm 0.004$ \\
\hline$g_{A i}\left(M_{Z}^{2}\right)$ & - & $-0.502 \pm 0.001$ \\
\hline$g_{v_{e}}\left(M_{Z}^{2}\right)$ & $-0.040_{-0.007}^{+0.008}$ & - \\
\hline$g_{A e}\left(M_{Z}^{2}\right)$ & $-0.503 \pm 0.002$ & - \\
\hline$g_{V \mu}\left(M_{Z}^{2}\right)$ & $-0.031_{-0.013}^{+0.011}$ & - \\
\hline$g_{A \mu}\left(M_{Z}^{2}\right)$ & $-0.501 \pm 0.002$ & - \\
\hline$g_{V \tau}\left(M_{Z}^{2}\right)$ & $-0.042_{-0.016}^{+0.013}$ & - \\
\hline$g_{A \tau}\left(M_{Z}^{2}\right)$ & $-0.502 \pm 0.003$ & - \\
\hline
\end{tabular}

Table 7: Derived quantities from fit results for all the ALEPH accumulated data $(1989,1990$, 1991 [1] and 1992).

quark charge asymmetry (1989-1990) [15] and $b$ and c forward-backward asymmetries (1990-1991) [16], the weak mixing angle becomes

$$
\sin ^{2} \theta_{e f f}^{\text {eept }}=0.2321 \pm 0.0012 \quad\left(\chi^{2} / \text { d.o.f. }=3.3 / 5\right)
$$

\section{Mass of the Top Quark}

The lineshape information and the effective weak mixing angle presented in the previous section can be used, within the context of the minimal Standard Model, to determine the top quark mass and the strong coupling constant, as presented in [1]. In this analysis $M_{H}$ is fixed at $300 \mathrm{GeV}$, and varied from $60 \mathrm{GeV}$ to $1000 \mathrm{GeV}$ to assess its effect on the uncertainty on the two parameters.

Fitting to the results for $M_{Z}, \Gamma_{Z}, R_{l}, \sigma_{h}^{0}, \sin ^{2} \theta_{e j f}^{d e p t}$ and the previously published measurement of 
Derived parameter No lepton univ.

$$
\begin{array}{ll}
g_{V_{e}}\left(M_{Z}^{2}\right) & -0.036 \pm 0.005 \\
g_{V_{\mu}}\left(M_{Z}^{2}\right) & -0.034 \pm 0.013 \\
g_{V_{\Gamma}}\left(M_{Z}^{2}\right) & -0.038 \pm 0.005
\end{array}
$$

Table 8: Effective vector coupling constants from fit results for all the ALEPH accumulated data (1989, 1990, 1991 [1] and 1992) using the added constraint of $\tau$ polarization.

$\Gamma_{b \bar{b}}[17]$ yields the top quark mass and the strong coupling constant:

$$
\begin{aligned}
m_{t} & =\left(184_{-29-18}^{+25+17}\right) \mathrm{GeV} \text { and } \\
\alpha_{s} & =0.113 \pm 0.011 \pm 0.002
\end{aligned}
$$

with $\chi^{2} /$ d.o.f. $=6.1 / 8$, where the second error in each reflects the uncertainty due to the Higgs mass. The theoretical uncertainties have been treated as in [18]. In particular, the uncertainty in the lowenergy hadronic part of the vacuum polarizations has been propagated in the fit and is included in the experimental error, which gets increased by about one $\mathrm{GeV}$ due to this. The probability contour is shown in fig. 5 . The ALEPH results can be combined with information on $M_{W}$ coming from neutrino experiments [19] and $\bar{p} p$ colliders [20]. In this case the result is

$$
\begin{aligned}
m_{t} & =\left(172_{-22-18}^{+20+17}\right) \mathrm{GeV} \text { and } \\
\alpha_{s} & =0.114 \pm 0.011 \pm 0.002
\end{aligned}
$$

with $\chi^{2} /$ d.o.f. $=7.3 / 11$. All results in sections 4 and 5 are in good agreement with the values published previously $[1,22]$.

\section{Limits on Extra Z Bosons}

As an example of the importance of the improved measurement of $\sigma_{h}^{0}$ in tests for departures from the Standard Model, a search for extra $Z$ bosons of one particular $E_{6}$-based model (the $\chi$-model [24]) is presented here.

After specifying the particular model (and without any assumption on the structure of the Higgs sector), there are only two free parameters

- The mixing angle between the symmetry eigenstates, $Z^{0}$ and $Z^{0 \prime}$, which will be called $\theta_{3}$.

- The mass of the heavier mass-eigenstate, that will be represented by $M_{Z^{\prime}}$.

Data taken at LEP are mainly sensitive to $\theta_{3}$ and only weakly to $M_{z^{\prime}}$, therefore only the $\theta_{3}$ determination is presented. The sensitivity to the mixing angle comes from the fact that the mixing between the two neutral bosons leads to changes in the effective fermion couplings to the lower mass boson, which are different for charged and neutral leptons and for up- and down-type quarks [24]. The uncertainty related to the lack of knowledge of $M_{Z}$, is included as part of the error in $\theta_{3}$. 


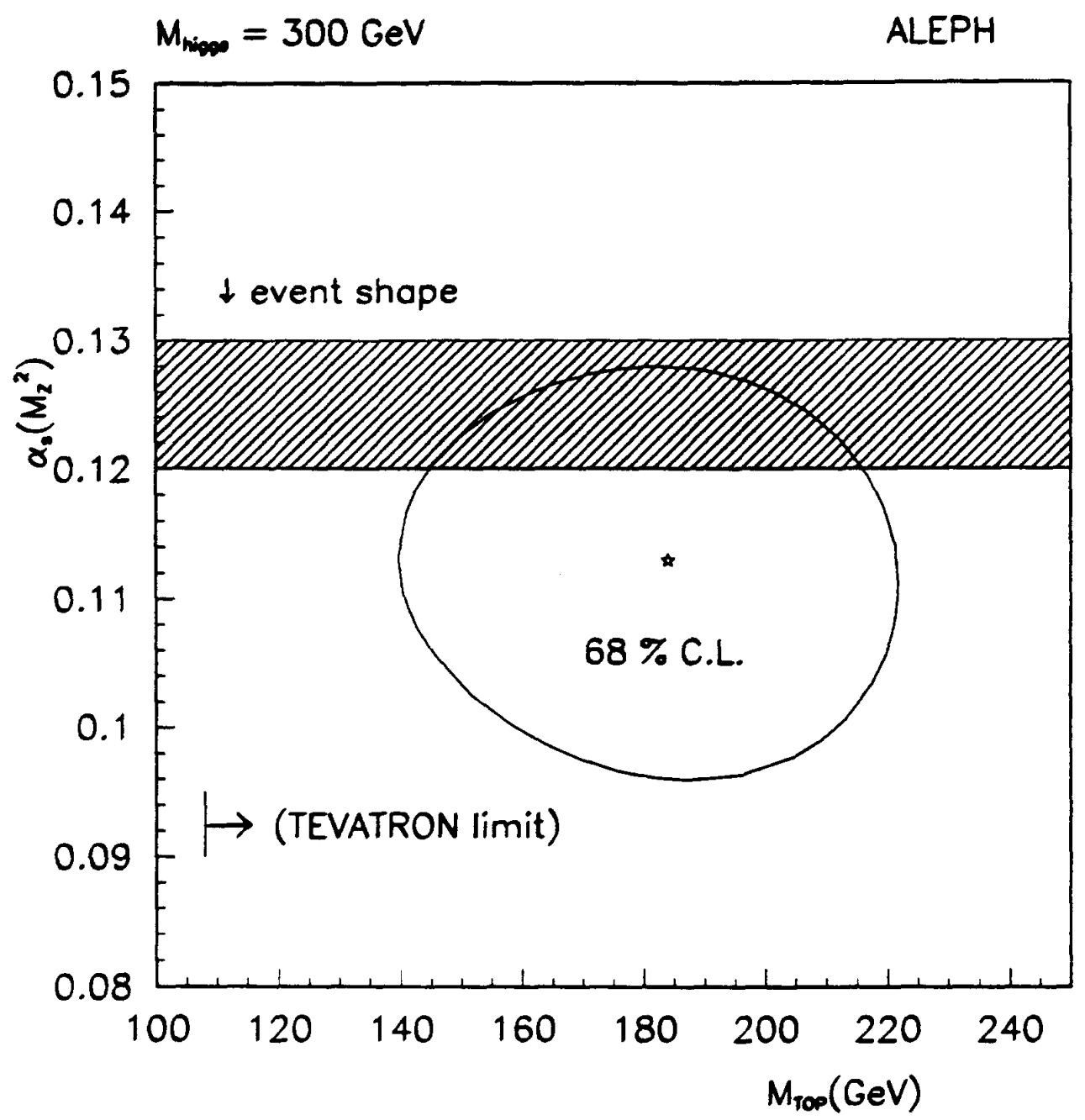

Figure 5: Contours of constant probability for the fit to the top quark mass, $m_{t}$, and the strong coupling constant, $\alpha_{s}$. The results of the event shape analysis [21] for $\alpha_{s}$ are shown by the hashed band. The Tevatron limit refers to the result of [23]. 
First, the entire lineshape and asymmetry data, parametrized in terms of $M_{Z}, \Gamma_{z}, R_{l}, \sigma_{h}^{0}$ and $A_{F B}^{0}$ are used together with a constraint on $\alpha_{0}$ from jet studies $\left(\alpha_{v}\left(M_{Z}^{2}\right)=0.125 \pm 0.005\right.$ [21]) to fit simultaneously for $M_{Z}, m_{t}, \alpha$, and $\theta_{3}$ using the program of [25]. The result is

$$
\theta_{3}=(-3.4 \pm 2.9) \cdot 10^{-3}
$$

for Higgs and $Z^{\prime}$ masses fixed at 300 and $500 \mathrm{GeV}$, respectively. Allowing the Higgs mass to vary from 60 to $1000 \mathrm{GeV}$ and the $Z^{\prime}$ mass from $320[26]$ to $1000 \mathrm{GeV}$, does not increase significantly the error on $\theta_{3}$. No deviation is seen from the minimal Standard Model prediction $\left(\theta_{3}=0\right)$, and a $95 \%$ confidence
level limit on the value of the mixing angle can be set to

$$
-0.0091<\theta_{3}<0.0023,
$$
which is significantly more stringent than those published using results prior to 1992 [27, 28]. A similar
conclusion is reached in [29] using preliminary 1992 results.

Second, in order to investigate the source of the sensitivity, a fit is made to get $M_{Z}, \alpha_{0}$ and $\theta_{3}$ using only the experimental values of $M_{Z}$ and $\sigma_{h}^{0}$ and the constraint on $\alpha_{a}$, while fixing the top quark mass to $150 \mathrm{GeV}$. The resulting uncertainty on $\theta_{3}$ is $\pm 3.3 \cdot 10^{-3}$, which increases to $\pm 3.4 \cdot 10^{-3}$ when the mentioned above is included. Reve between 100 and $200 \mathrm{GeV}$. The variation in Higgs and $\mathrm{Z}^{\prime}$ masses rest unchanged, increases the error to $\pm 5.8 \cdot 10^{-3}$, peak cross section from the first fit, leaving the constrained) comes from the measurement of $\sigma^{0}$, which implies that most of the sensitivity (with $\alpha_{s}$ in determining the mixing angle; removing it ${ }_{h}$. On the other hand, the constraint on $\alpha$, is also crucial two.

The qualitative picture deduced from these results does not change if different $E_{6}$ related models are chosen. The hadronic peak cross section, in conjunction with an external constraint on the strong coupling constant, is a powerful variable when testing for signals of extra neutral bosons.

\section{Conclusion}

In addition to a substantial increase in the data at the peak of the $Z$ resonace, th in the luminosity measurement due to the new silicon peak of the $Z$ resonance, the increased precision ments in the understanding of the hadron selection actungsten luminosity monitor, as well as improvesubstantially reduced previous uncertainties in the paccuracy and the tau-pair selection algorithm, have parameters of the $\mathrm{Z}$ resonance, especially the hadronic neutrino families is found to be $N_{\nu}=2.983 \pm 0.034$, , the effective weak mixing angle determined from the $\theta_{\text {eff }}^{\text {eept }}=0.2310 \pm 0.0019$. Including the previous ALEPH plarization asymmetry, the quark charge asymmetry, and $b$ including the results from $\Gamma_{b \bar{b}}$, is $m_{t}=\left(184^{2} \theta_{e f f}=0.2321 \pm 0.0012\right.$. The top quark mass in this frame,

\section{Acknowledgements}

It is a pleasure to thank our colleagues from the accelerator divisions for the successful operation of the machine. We are indebted to the engineers and technicians in all our institutions for their contributions 
to the successful performance of ALEPH. Those of us from non-member countries thank CERN for its hospitality.

\section{References}

[1] D. Buskulic et al., ALEPH Collab., Z. Phys. C60 (1993) 71.

[2] D. Decamp et al., ALEPH Collab., Z. Phys. C53 (1992) 1;

D. Decamp et al., ALEPH Collab., Z. Phys. C48 (1990) 365.

[3] D. Decamp et al., ALEPH Collab., Nuclear Instruments and Methods A294 (1990) 121.

[4] D. Bédérède et al., SiCAL - A High Precision Silicon-Tungsten Luminosity Calorimeter for ALEPH, paper in preparation for Nuclear Instruments and Methods.

[5] L. Arnaudon et al., The Energy Calibration of LEP in 1992, CERN-SL/93-21 (DI) 1993.

[6] D. Decamp et al., ALEPH Collab., Z. Phys. C53 (1992) 375.

[7] S. Jadach, E. Richter-Was, Z. Was and B.F.L. Ward, Phys. Lett. B268 (1991) 253;

S. Jadach, E. Richter-Wa̧s, Z. Wąs and B.F.L. Ward, Comput. Phys. Commun. 70 (1992) 305;

S. Jadach and B.F.L. Ward, Phys. Rev. D40 (1989) 3582.

[8] M. Böhm, A. Denner and W. Hollik. Nucl. Phys. B304 (1988) 687;

F.A. Berends, R. Kleiss and W. Hollik. Nucl. Phys. B304 (1988) 712.

[9] W. Beenakker and B. Pietrzyk, Phys. Lett. B304 (1993) 366; W. Beenakker and B. Pietrzyk, Phys. Lett. B296 (1992) 296.

[10] W. Beenakker, F. A. Berends and S. C. van der Marck, Nucl. Phys. B349 (1991) 323.

[11] D. Decamp et al., ALEPH Collab., Phys. Rep. 216 (1992) 253.

[12] M. Martinez, L. Garrido, R. Miquel, J.L. Harton and R. Tanaka, Z. Phys. C49 (1991) 645.

[13] B. A. Kniehl and A. Sirlin, Phys. Rev. D47 (1993) 883;

S. Fanchiotti, B. A. Kniehl and A. Sirlin, Phys. Rev. D48 (1993) 307;

R. Barbieri et al., Phys. Lett. B288 (1992) 95;

K. G. Chetyrkin, J. H. Kühn, Phys. Lett. B248 (1990) 359;

K. G. Chetyrkin, J. H. Kühn and A. Kwiatkowski, Phys. Lett. B282 (1992) 221;

J. Fleischer, O. V. Tarasov and F. Jegerlehner, Phys. Lett. B293 (1992) 437.

[14] D. Buskulic et al., ALEPH Coll., Z. Phys. C59 (1993) 369.

[15] D. Decamp et al., ALEPH Coll., Phys. Lett. B259 (1991) 377.

[16] D. Buskulic et al., ALEPH Coll., CERN-PPE/94-17.

[17] D. Buskulic et al., ALEPH Coll., Phys. Lett. B313 (1993) 535; Phys. Lett. B313 (1993) 549.

[18] The LEP collaborations, Updated Parameters of the $Z^{0}$ Resonance from Combined Preliminary Data of the LEP Experiments, CERN/PPE/93-157 (26 August 1993). 
[19] C. G. Arroyo et al., CCFR Coll., Columbia University preprint NEVIS R\#1498, November 1993. H. Abramowicz et al., CDHS Coll., Phys. Rev. Lett. 57 (1986) 298; A. Blondel et al., Z. Phys. C45 (1990) 361.

J. V. Allaby et al., CHARM Coll., Phys. Lett. B177 (1986) 446; Z. Phys. C36 (1987) 611.

[20] J. Alitti et al., UA2 Coll., Phys. Lett. B241 (1990) 150.

F. Abe et al., CDF Coll., Phys. Rev. Lett. 65 (1990) 2243.

[21] D. Decamp at al., ALEPH Coll., Phys. Lett. B284 (1992) 163.

[22] P. Abreu et al., DELPHI Coll., CERN-PPE/94-08.

O. Adriani et al., L3 Coll., Phys. Rep. 236 (1993) 1.

R. Akers et al., OPAL Coll., CERN-PPE/93-146.

[23] A. Barbiero-Galtieri, plenary talk at the EPS Conference, Marseille, July 1993. Recently, the D0 collaboration has reported a more stringent limit on the top quark mass in S. Abachi et al., D0 Coll., FERMILAB-PUB-94-004-E.

[24] R. W. Robinett, Phys. Rev D26 (1982) 2388;

R. W. Robinett and J. L. Rosner, Phys. Rev D26 (1982) 2396;

F. del Aguila, G. Blair, M. Daniel, G. G. Ross, Nucl. Phys. B272 (1986) 413;

J .L. Hewett, T. G. Rizzo, J. A. Robinson, Phys. Rev. D33 (1986) 1476; ibid. D34 (1986) 2179;

D. London and J. L. Rosner, Phys. Rep. 34 (1986) 1530;

P. Binetruy, S. Dawson, T. Hinchliffe, M. Sher, Nucl. Phys. B273 (1986) 501;

J. Ellis, K. Enquist, D. V. Nanopoulos, F. Zwirner, Nucl. Phys. B276 (1986) 14.

G. Belanger and S. Godfrey, Phys. Rev. D35 (1987) 378.

[25] A. Leike, S. Riemann and T. Riemann, University of Munich preprint LMU-91/06, and FORTRAN program ZEFIT;

A. Leike, S. Riemann and T. Riemann, Phys. Lett. B291 (1992) 187.

[26] F. Abe et al., CDF Collab., Phys. Rev. Lett. 68 (1992) 1463.

[27] O. Adriani et al., L3 Collab., Phys. Lett. B306 (1993) 187.

[28] P. Langacker and M. Luo, Phys. Rev. D45 (1992) 278;

E. Nardi, E. Roulet and D. Tommasini, Phys. Rev. D46 (1992) 3040;

F. del Aguila, W. Hollik, J. M. Moreno and M. Quiros, Nucl. Phys. B372 (1992) 1;

M. C. Gonzalez-Garcia and J. W. F. Valle, Phys. Lett. B259 (1991) 365;

G. Bhattacharyya et al., Mod. Phys. Lett. A6 (1991) 2552.

[29] G. Altarelli et al., Phys. Lett. B318 (1993) 139. 


\section{A Appendix}

A.1 Correlation Matrix for 5-Parameter Fit

\begin{tabular}{ccccc}
\hline & $\Gamma_{z}$ & $\sigma_{h}^{0}$ & $\mathrm{R}_{\mathrm{l}}$ & $A_{F B}^{0, l}$ \\
& & & & \\
\hline $\mathrm{M}_{z}$ & 0.017 & 0.022 & -0.001 & 0.175 \\
$\Gamma_{z}$ & - & -0.127 & 0.011 & 0.008 \\
$\sigma_{h}^{0}$ & & - & 0.216 & 0.009 \\
$\mathrm{R}_{l}$ & & & - & 0.007 \\
\hline
\end{tabular}

A.2 Correlation Matrix for 9-Parameter Fit

\begin{tabular}{|c|c|c|c|c|c|c|c|c|}
\hline & $\Gamma_{z}$ & $\sigma_{h}^{0}$ & $\mathrm{Re}_{e}$ & $R_{\mu}$ & $\mathrm{R}_{\tau}$ & $A_{F B}^{0, e}$ & $A_{F B}^{0, \mu}$ & $A_{F B}^{0, \tau}$ \\
\hline $\mathrm{M}_{Z}$ & 0.014 & 0.019 & -0.002 & 0.002 & -0.001 & 0.076 & 0.102 & 0.086 \\
\hline$\Gamma_{z}$ & - & -0.127 & 0.002 & 0.018 & 0.001 & 0.005 & 0.005 & 0.004 \\
\hline$\sigma_{h}^{0}$ & & - & 0.137 & 0.133 & 0.130 & 0.005 & 0.006 & 0.005 \\
\hline $\mathrm{R}_{e}$ & & & - & 0.078 & 0.072 & 0.005 & 0.000 & 0.000 \\
\hline $\mathrm{R}_{\mu}$ & & & & - & 0.071 & 0.001 & 0.009 & 0.000 \\
\hline$R_{\tau}$ & & & & & - & 0.000 & 0.000 & 0.007 \\
\hline$A_{F B}^{0, e}$ & & & & & & - & 0.042 & 0.039 \\
\hline$A_{F B}^{0, \mu}$ & & & & & & & - & 0.040 \\
\hline
\end{tabular}

